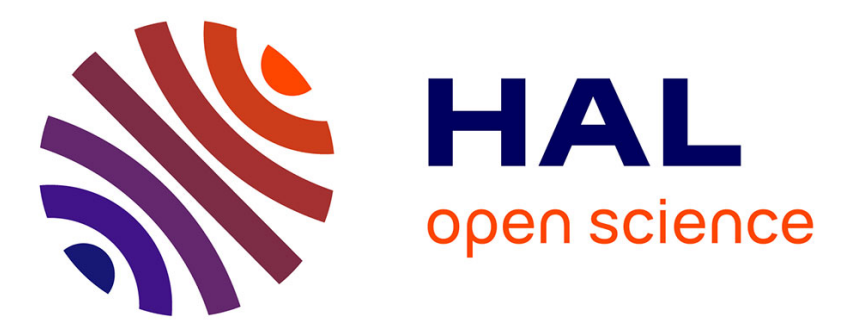

\title{
Optimization of bandpass optical filters based on $\mathrm{TiO} 2$ nanolayers
}

\author{
Nathalie Démarest, Damien Deubel, Jean-Claude Keromnès, Claude Vaudry, \\ Fabien Grasset, Ronan Lefort, Maryline Guilloux-Viry
}

\section{- To cite this version:}

Nathalie Démarest, Damien Deubel, Jean-Claude Keromnès, Claude Vaudry, Fabien Grasset, et al.. Optimization of bandpass optical filters based on TiO2 nanolayers. Optical Engineering, 2015, 54 (1), pp.015101. 10.1117/1.OE.54.1.015101 . hal-01099437

\section{HAL Id: hal-01099437 https://hal.science/hal-01099437}

Submitted on 3 Jan 2015

HAL is a multi-disciplinary open access archive for the deposit and dissemination of scientific research documents, whether they are published or not. The documents may come from teaching and research institutions in France or abroad, or from public or private research centers.
L'archive ouverte pluridisciplinaire HAL, est destinée au dépôt et à la diffusion de documents scientifiques de niveau recherche, publiés ou non, émanant des établissements d'enseignement et de recherche français ou étrangers, des laboratoires publics ou privés. 


\section{Optical Engineering}

\section{Optimization of bandpass optical filters based on $\mathrm{TiO}_{2}$ nanolayers}

Nathalie Démarest

Damien Deubel

Jean-Claude Keromnès

Claude Vaudry

Fabien Grasset

Ronan Lefort

Maryline Guilloux-Viry 


\title{
Optimization of bandpass optical filters based on $\mathrm{TiO}_{2}$ nanolayers
}

\author{
Nathalie Démarest, ${ }^{\mathrm{a}, \mathrm{b}}$ Damien Deubel, ${ }^{\mathrm{a}}$ Jean-Claude Keromnès, ${ }^{\mathrm{a}}$ Claude Vaudry, ${ }^{\mathrm{a}}$ Fabien Grasset, ${ }^{\mathrm{b}, \mathrm{c}, *}$ Ronan Lefort, ${ }^{\mathrm{d}, \star}$ and \\ Maryline Guilloux-Viry \\ aKerdry Thin Solid Films, 5 rue De Broglie, 22300 Lannion, France \\ 'Institut des Sciences Chimiques de Rennes, UMR 6226 UR1-CNRS, Université de Rennes 1, Campus de Beaulieu, \\ 35042 Rennes Cedex, France \\ 'National Institute of Material Science, CNRS, UMI 3629, Laboratory for Innovative Key Materials and Structures (LINK), 1-1 Namiki, 305-0044 \\ Tsukuba, Japan \\ IInstitut de Physique de Rennes, 263 Avenue du General Leclerc CS 74205, 35042 Rennes Cedex, France
}

\begin{abstract}
The design and realization of high-quality bandpass optical filters are often very difficult tasks due to the strong correlation of the optical index of dielectric thin films to their final thickness, as observed in many industrial deposition processes. We report on the optimization of complex optical filters in the visible and NIR spectral ranges as realized by ion beam-assisted electron beam deposition of silica and titanium oxide multilayers. We show that this process always leads to amorphous films prior to thermal annealing. On the contrary, the optical dispersion of $\mathrm{TiO}_{2}$ nanolayers is highly dependent on their thickness, while this dependence vanishes for layers thicker than $100 \mathrm{~nm}$. We demonstrate that accounting for this nonlinear dependence of the optical index is both very important and necessary in order to obtain high-quality optical filters. $\odot 2015$ Society of Photo-Optical Instrumentation Engineers (SPIE) [DOI: 10.1117/1.OE.54.1.015101]
\end{abstract}

Keywords: titanium dioxide; thin film; spectroscopic ellipsometry; optical properties; e-beam evaporation.

Paper 141335 received Aug. 25, 2014; accepted for publication Nov. 25, 2014; published online Jan. 2, 2015.

\section{Introduction}

The design of high-quality bandpass optical filters requires the association of materials with a high-optical index contrast, alternated in a complex series of thin layers, usually with thicknesses ranging from 10 to $1000 \mathrm{~nm} \cdot{ }^{1-3}$ Although the design of such optical devices is now eased by efficient numerical computation tools ${ }^{4,5}$ the realization of such multilayered dielectric heterostructures remains an important technological challenge.

For filters transparent in the visible and near-infrared (NIR) spectral regions, the highly contrasted titanium oxide (high index)/silicon oxide (low index) has been extensively studied. ${ }^{6} \mathrm{TiO}_{2}$ is widely encountered in sensors, antireflection coatings, or photocatalysis applications. ${ }^{7-9} \mathrm{TiO}_{2}$ is a large bandgap semiconductor and possesses an exceptional chemical stability. For these reasons, it is an important candidate for thin film multilayer dielectric coatings designed for high reflectivity mirrors, dichroic filters, and so on.

Many techniques have been proposed for the preparation of $\mathrm{TiO}_{2}$ thin films so far. These include electron-beam evaporation, ${ }^{10}$ radio frequency magnetron sputtering, ${ }^{11}$ direct current magnetron sputtering, ${ }^{12}$ pulsed laser deposition, ${ }^{13}$ chemical vapor deposition, ${ }^{14}$ atomic layer deposition, ${ }^{15}$ sol-gel chemistry, ${ }^{14,16}$ and so on. It has been shown that the different physical and chemical conditions imposed on the material during these processes can strongly influence a range of optical properties for the final deposited film. ${ }^{17-19}$ In order to understand and then finely design the optical properties of $\mathrm{TiO}_{2}$ thin films, it is of upmost importance to control their thermodynamic state (amorphous, anatase,

*Address all correspondence to: F. Grasset, E-mail: Fabien.Grasset@univrennes1.fr; R. Lefort, E-mail: Ronan.Lefort@univ-rennes1.fr or rutile crystal phase), their microstructure (nanostructure, porosity, anisotropic texture, alignment on the substrate, and rugosity), and homogeneity. Any deviation from standardized process parameters could indeed lead to light diffusion or optical index mismatch incompatible with the design of high-quality complex optical filters. ${ }^{20}$ In particular, it has been shown several times that in many processes, the final optical dispersion of the $\mathrm{TiO}_{2}$ thin films was dependent on the layer thickness. ${ }^{14,21-23}$

In the present paper, we report on the optimization of an industrial ion-assisted deposition (IAD) process ${ }^{10,18,24}$ of $\mathrm{TiO}_{2}$ thin films in the range of thicknesses varying from 15 to $500 \mathrm{~nm}$, targeting the realization of high-quality complex bandpass optical filters in the visible and NIR spectral ranges. The physical properties of the as-deposited (nonannealed) thin films are studied by $\mathrm{x}$-ray diffraction and scanning electron microscopy (SEM), and the optical dispersions were studied by variable angle spectroscopic ellipsometry (VASE).

\section{Material and Methods}

\subsection{Evaporation of $\mathrm{TiO}_{2}$ Films}

The substrates used were borosilicate (BK7) glasses. The substrates were cleaned with soap solution, rinsed in deionized water, and dried under nitrogen gas flow. In the Leybold LH1100 chamber, a background pressure of $3 \times 10^{-6} \mathrm{mbar}$ was reached using a mechanical pump (CIT Alcatel) and a cyopump (Leybold coolVac 10.000). $\mathrm{TiO}_{2}$ films were ebeam deposited with an operating pressure of $2.0 \times 10^{-4}$ mbar in a reactive oxygen atmosphere at a constant flow

0091-3286/2015/\$25.00 @ 2015 SPIE 
of $30 \mathrm{sccm}$, monitored by MKS mass flow controller. The 99.9\% pure $\mathrm{TiO}_{2}$ granulates (Umicore) were used as a source material. Ion assistance is performed by injecting pure oxygen and pure argon straight into the source (Ions tech, 16-cm RF). Films (from 15- to 500-nm thick) were deposited at a speed of $3 \AA / s$. The rate was controlled by a quartz balance and the thickness of the layers was controlled by in situ optical monitoring (except for the values extracted from ellipsometric results, all thicknesses mentioned are derived from this in situ control).

\subsection{Experimental Details}

Surface and cross-section morphologies of as-deposited $\mathrm{TiO}_{2}$ films were observed by SEM using a JEOL (JSM $6301 F)$ microscope. Surface roughness was measured with an atomic force microscope (AFM) D3100 with nanoscope $\mathrm{V}$ (Bruker). The structure of the films was analyzed by an $\mathrm{X}$-ray diffraction using a Bruker D8 advance diffractometer in a theta-2theta Bragg-Brentano geometry.

The optical dispersions of the thin films were analyzed by VASE on a Horiba-Jobin Yvon UVISEL ellipsometer equipped with a phase modulation detection. In order to avoid incoherent reflection from the BK7 substrate, the back side of each sample glass plate has been depolished with abrasive paper. The measurement then consists of the acquisition of the complex ratio of reflexion coefficients of the $p$ and $s$ polarization components of the light beam: ${ }^{25}$

$\rho=r_{p} / r_{s}=\left(E_{r p} / E_{i p}\right) /\left(E_{r s} / E_{i s}\right)=\tan \Psi e^{j \Delta}$.

The ellipsometric composite quantities $I_{\mathrm{S}}$ and $I_{\mathrm{C}}$ $\left[I_{\mathrm{S}}=\sin (2 \Psi) \sin \Delta\right.$ and $I_{\mathrm{C}}=\sin (2 \Psi) \cos \Delta$ for a standard hardware configuration with polarizer oriented at $45 \mathrm{deg}$ and analyzer oriented at $0 \mathrm{deg}$ ] were measured over the spectral range from 300 to $2000 \mathrm{~nm}$ for three different incidence angles (50 deg, $60 \mathrm{deg}$, and $70 \mathrm{deg}$ ) for each sample. Then two ellipsometric angles $\Psi$ and $\Delta$ are deduced from $I_{\mathrm{S}}$ and $I_{\mathrm{C}}$, and were then fitted with an appropriate model using a standard least squares $\left(\chi^{2}\right)$ algorithm as provided by the Horiba Delta Psi2 software (in order to prevent the limited indetermination on the values of $\Psi$ and $\Delta$ arising from the inversion of the trigonometric relations with $I_{\mathrm{S}}$ and $I_{\mathrm{C}}$, the data have been consolidated by merging measurements done with two different hardware configurations).

\section{Results}

\subsection{X-Ray Diffraction}

Figure 1 shows a typical diffraction pattern of an as-deposited (nonannealed) $\mathrm{TiO}_{2}$ film (thickness of $300 \mathrm{~nm}$ ) compared with that of the BK7 substrate. Clearly, no extra peak is found in addition to the amorphous halo of the substrate, indicating the amorphous nature of the $\mathrm{TiO}_{2}$ thin films. The same result is found whatever the film thickness (10 to $500 \mathrm{~nm})$. Although already reported in other works, ${ }^{26}$ this result contrasts with other reported observations (through different deposition processes or thermal treatments) that report the often predominance of the polycrystalline anatase phase of $\mathrm{TiO}_{2} \cdot{ }^{10,22,27,28}$ The classical relevance of an annealing isothermal step in the process in favor of the anatase or rutile phases growth has been underlined. ${ }^{10,18,26}$

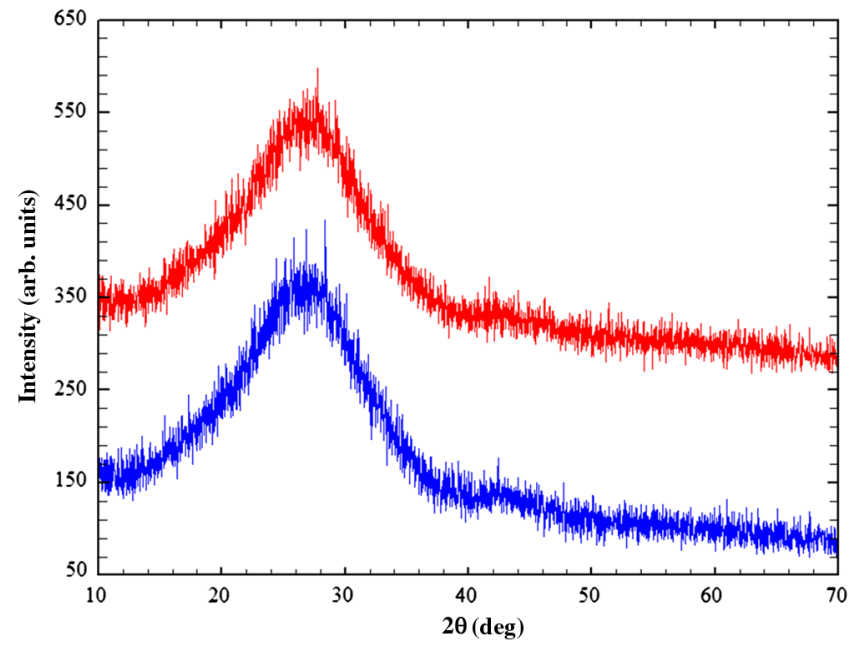

Fig. 1 Diffractograms of the glass substrate (lower curve) and of $\mathrm{TiO}_{2}$ (monolayer with 300-nm thickness on glass substrate; upper curve).

\subsection{Atomic Force and Scanning Electron Microscopies}

The surface morphologies of $\mathrm{TiO}_{2}$ films were observed by AFM (tapping mode). One of the $300 \mathrm{~nm}$ film is shown in Fig. 2. The root mean square value of the surface roughness was $0.6 \mathrm{~nm}$ within a $1 \mu \mathrm{m} \times 1 \mu \mathrm{m}$ scan regardless of the layer thickness. This is the same value for the borosilicate glass without coating. This low value obtained by IAD is interestingly much better than some others reported for other methods. ${ }^{20,23}$

The SEM characterization allows for the observation of the surface state of $\mathrm{TiO}_{2}$ monolayers (Fig. 3). The surface state of layer (the chosen example is the 300 -nm layer) is really homogenous and has a very low number of defects as observed in the SEM figures (low and high magnifications).

While most of the surface inclusion can be safely classified as defects, some nodule-shaped inclusions deserve more attention (see Fig. 4). They can be tentatively assigned to anatase nucleation sites, as already reported elsewhere. ${ }^{20}$ The number of nodules is very low thanks to the additional energy of the ions which may suppress the nodule growth. ${ }^{20}$

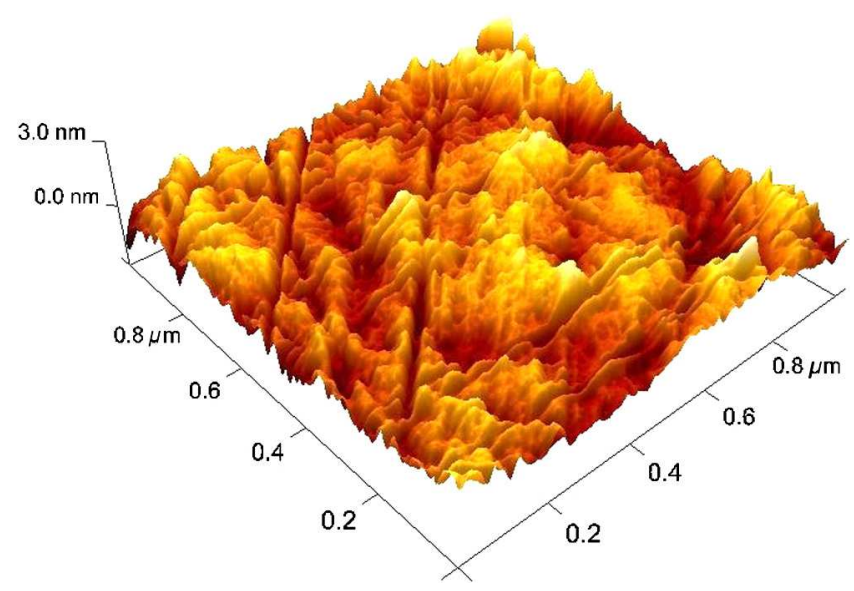

Fig. 2 Atomic force microscope (AFM) image of 300-nm $\mathrm{TiO}_{2}$ film. Root mean square calculated is $0.6 \mathrm{~nm}$ for all layers and glass substrate. 


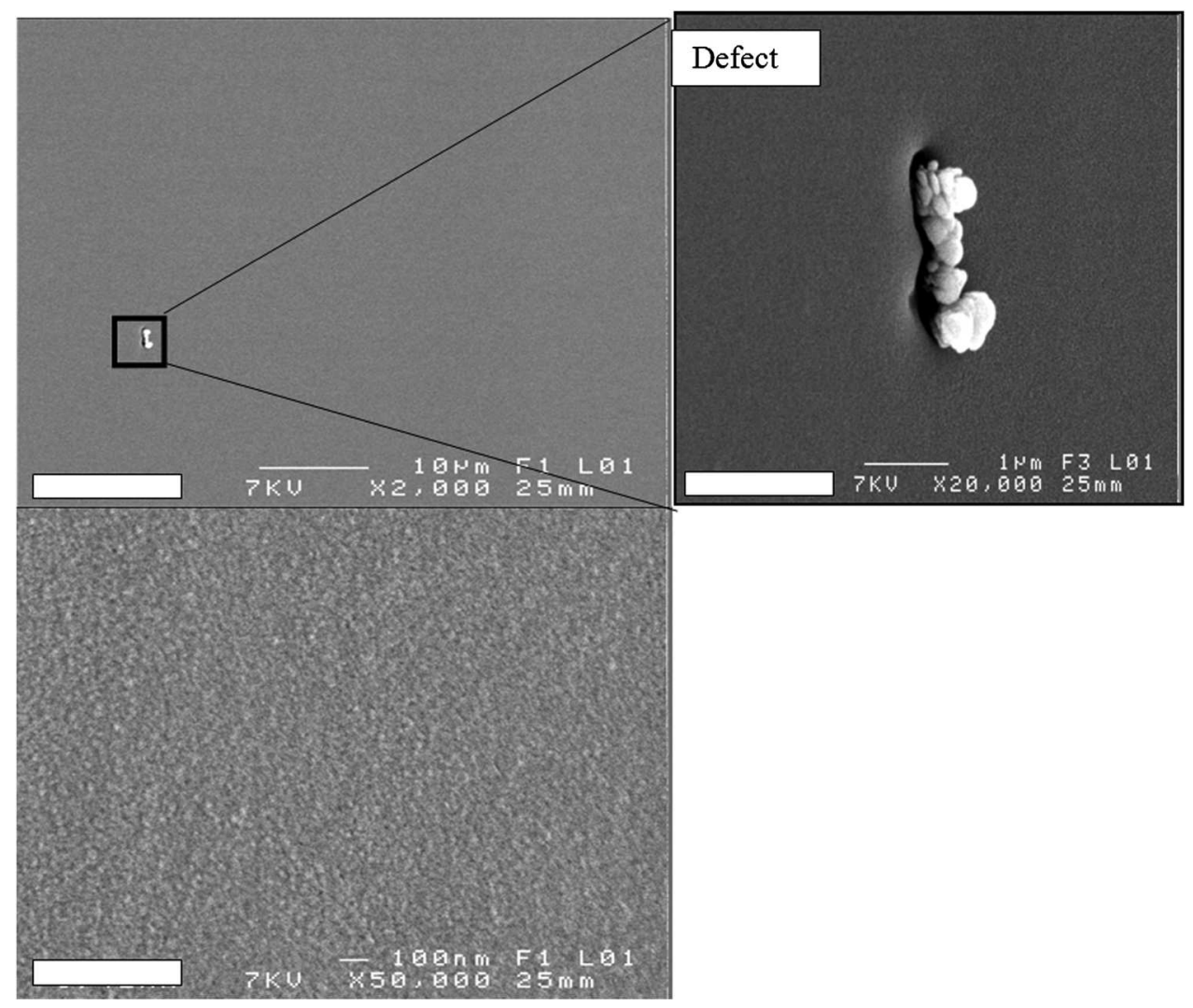

Fig. 3 Scanning electron microscopy (SEM) images of the surface of $300-\mathrm{nm} \mathrm{TiO}_{2}$ films at low and high magnifications.

\subsection{Ellipsometric Results}

The ellipsometric angles $\Psi$ and $\Delta$ measured on 30- and 300-nm thick $\mathrm{TiO}_{2}$ layers deposited on $\mathrm{BK} 7$ substrates are presented in Fig. 5.

For the 30-nm thin film, these data show the general features (low $\Psi$ values, $\Delta$ between $200 \mathrm{deg}$ and $300 \mathrm{deg}$ ) of a system, transparent in the visible and NIR regions. These properties are reminiscent of the glass substrate properties and suggest that the deposited film brings neither strong optical absorption nor strong metallic reflection, as expected for a high energy gap $\mathrm{TiO}_{2}$ thin film. For the 300 -nm film, interference oscillations are clearly visible due to the closing of the film thickness to the wavelength.

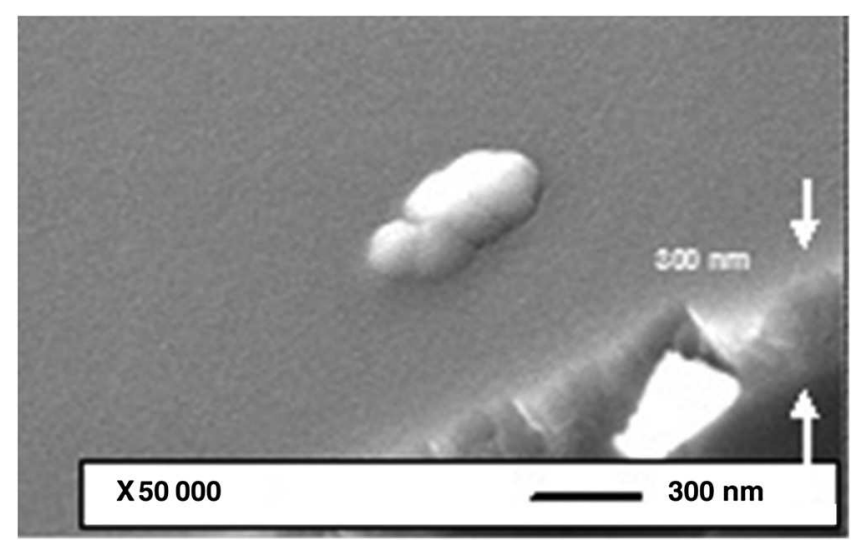

Fig. 4 SEM images of 300-nm thick $\mathrm{TiO}_{2}$ film.
In order to extract meaningful information from these data, it is necessary to compare them to a theoretical model that includes assumptions on (1) the general sample layer structure, (2) the layers thicknesses, and (3) the optical dispersions of the substrate and of each layer. In the present case, the simplest sample structure was assumed: a dense $\mathrm{TiO}_{2}$ layer of thickness $e$ sitting on top of a semi-infinite BK7 substrate. Two other more complex models were used in comparison (1) with an inhomogeneous single layer (represented by a mixture of dense material and void) and (2) a double-layer film with a dense $\mathrm{TiO}_{2}$ layer above an inhomogeneous, ${ }^{4}$ but no significant improvements were obtained. The rugosity of the samples was classically described as a supplementary layer of thickness $e_{\mathrm{r}}$ (Fig. 6).

The optical parameters of each layer are completely described by the complex dispersion curve $\underline{n}(\lambda)=n(\lambda)-$ $i \kappa(\lambda)$, where $n(\lambda)$ is the usual optical index and $\kappa(\lambda)$ is the extinction coefficient. The dispersion curve of the BK7 substrate was determined experimentally on a bare glass plate. These experimental data were fitted by a classical Cauchy law that was used afterward for all other samples to describe the substrate optical properties without further refinement (used as fixed parameters).

In the literature, the optical dispersion of $\mathrm{TiO}_{2}$ has been modeled by various theoretical expressions, among which Cauchy, Forouhi-Bloomer, Cody-Lorentz, or Tauc-Lorentz models are most often encountered. ${ }^{10,21,27,28}$ In the present work, the $\mathrm{TiO}_{2}$ layers were described using the TaucLorentz model, ${ }^{29}$ which combines Tauc's description of interband transitions above the band edge with the Lorentz's classical oscillator model. In parallel, the optical properties 

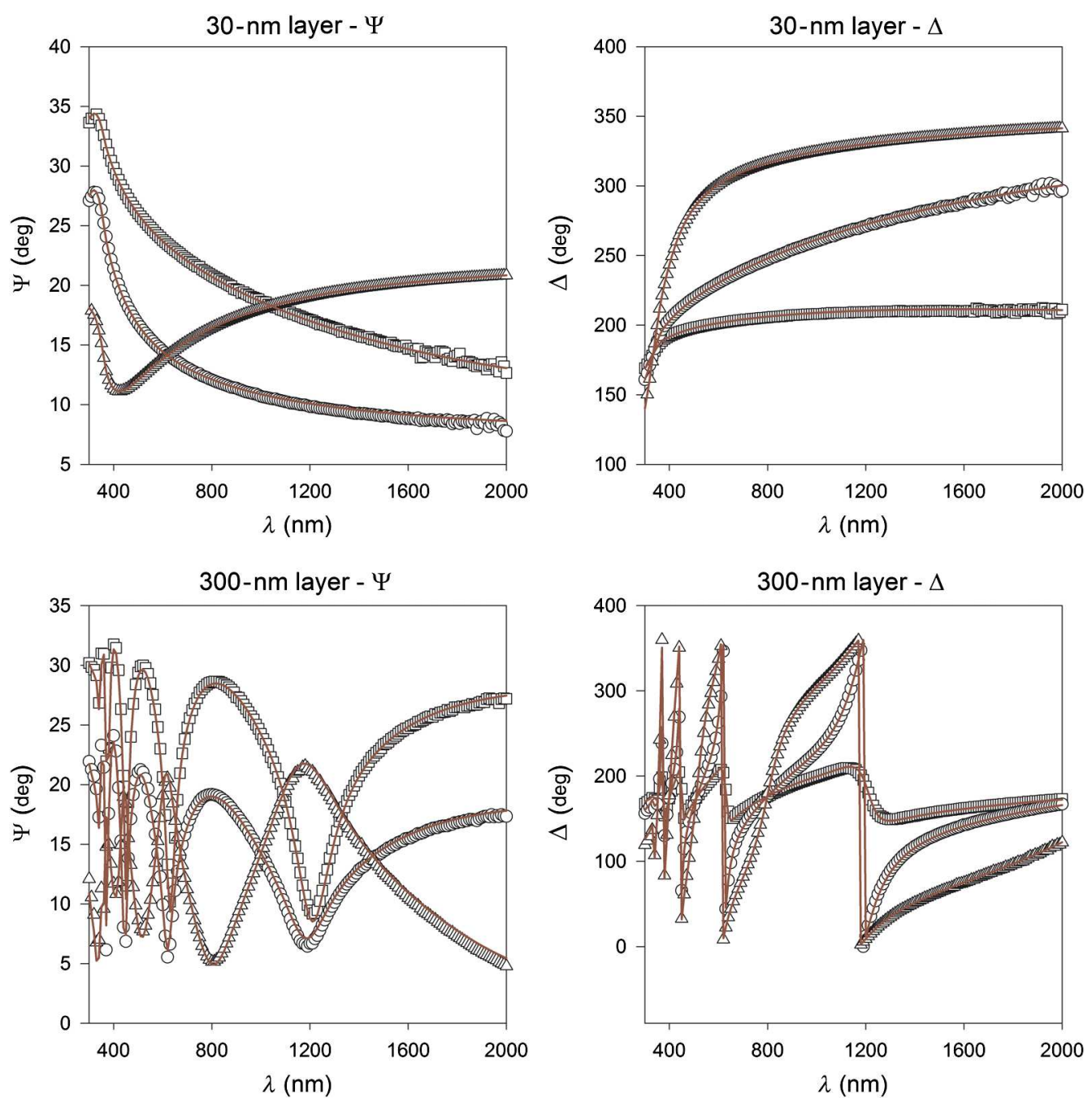

Fig. 5 Ellipsometric angles measured on 30- and 300-nm thick $\mathrm{TiO}_{2}$ layers on $\mathrm{BK} 7$ depolished substrates, measured with incidence angles of $50 \mathrm{deg}$ (squares), $60 \mathrm{deg}$ (circles), and $70 \mathrm{deg}$ (triangles). The solid lines are best fits using the model detailed in the text.

of the $\mathrm{TiO}_{2}$ films were described by two different dispersion models depending on the wavelength: the transparent regime (450 to $1400 \mathrm{~nm}$ ) with Cauchy absorbent dispersion and the absorbing regime (300 to $450 \mathrm{~nm}$ ) with ForouhiBloomer dispersion, ${ }^{4}$ but again, no significant improvements were obtained. Moreover, the Tauc-Lorentz dispersion is considered as particularly well suited for representing amorphous semiconducting materials.

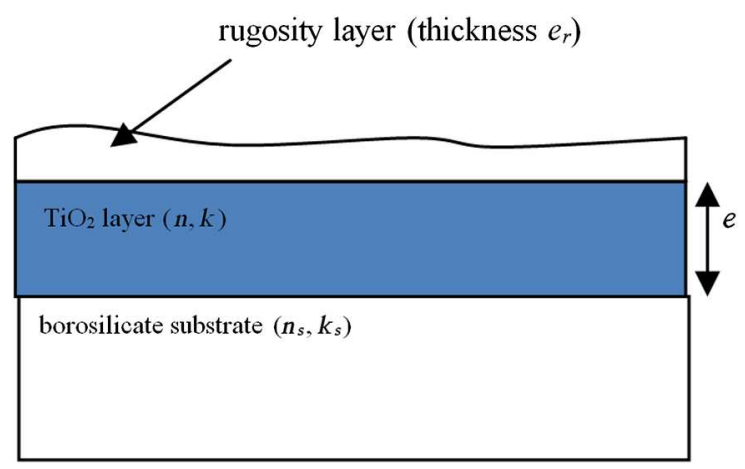

Fig. 6 Sample layered structure.
The Tauc-Lorentz dispersion can be expressed in terms of the imaginary part of the dielectric function of the material: ${ }^{29}$

$\varepsilon^{\prime \prime}(E)= \begin{cases}\frac{1}{E} \frac{A E_{0} C\left(E-E_{\mathrm{g}}\right)^{2}}{\left(E^{2}-E_{0}^{2}\right)^{2}+C^{2} E^{2}} & \text { for } E>E_{\mathrm{g}} \\ 0 & \text { for } E \leq E_{\mathrm{g}}\end{cases}$

from which the real part $\varepsilon^{\prime}(E)=\varepsilon_{\infty}+\varepsilon_{r}(E)$ can be derived through Kramers-Kronig integration. A total of five parameters, namely the high-frequency dielectric constant $\varepsilon_{\infty}$, the optical bandgap $E_{\mathrm{g}}$, an amplitude coefficient $A$, the oscillator peak energy $E_{0}$, and linewidth $C$, have to be refined during a fitting procedure aiming to compare with the experimental data. In addition to these five optical quantities, the thickness of the $\mathrm{TiO}_{2}$ layer was also considered as a free parameter.

Except for the 18-nm layer, a rugosity top layer was needed in order to achieve a satisfactory agreement with the experiment. In order to reduce the total number of free parameters, this layer was classically described as a 50:50 mixture of $\mathrm{TiO}_{2}$ and void, calculating the resulting index in the frame of the Bruggeman effective medium approximation (using for the $50 \% \mathrm{TiO}_{2}$ part the same dispersion parameters as the dense $\mathrm{TiO}_{2}$ layer). Also, after a few preliminary tests, a 

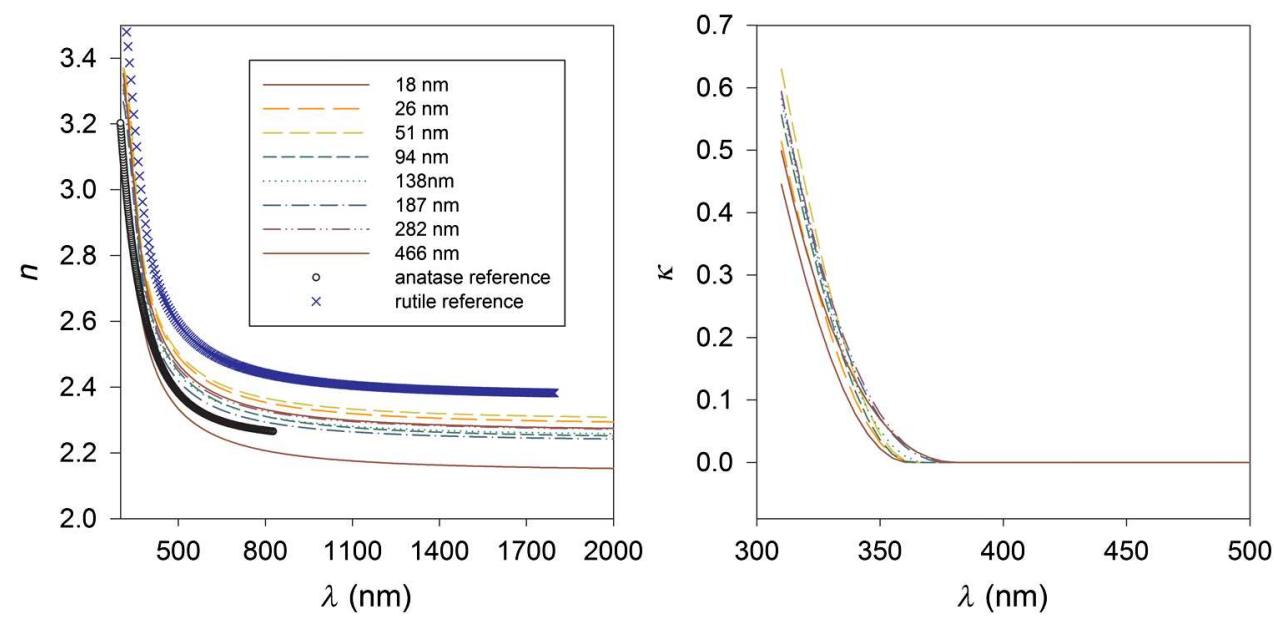

Fig. 7 Complex optical dispersion curves of the different $\mathrm{TiO}_{2}$ layers as computed from the best fits, and compared to the reference dispersions of crystalline anatase and rutile.

constant thickness of $2.8 \mathrm{~nm}$ was used as a fixed parameter for all samples thicker than $18 \mathrm{~nm}$. This low value describing the rugosity of the samples agrees semiquantitatively with the AFM results presented above and suggests that the IAD process used here does not induce any additional rugosity to the deposited layers than that imposed by the substrate, even for thick $\mathrm{TiO}_{2}$ films.

The model described above was fitted using a standard least-squares procedure to the experimental ellipsometric angles. In particular, the data of the three incident angles of the VASE experiment were used simultaneously to refine a single set of six free parameters per sample. Even if the sample structure proposed here is probably oversimplified and cannot account for all physical properties of the real samples, this few parameters procedure is very selective compared with other approaches based on more complicated sample structures often proposed in the literature..$^{10,27,28}$ It is, therefore, a severe test of the reliability of the physical quantities that can be extracted from the experiment.

The solid lines presented in Fig. 7 are the best fits of the described model to the experimental data. Clearly, the agreement is excellent in both the transparency and absorption spectral ranges. The fitted layers' thicknesses are comparable with the ones targeted in the IAD process within a precision better that $10 \%$.

Within the six free parameters for each sample, only the amplitude term $A$, and to a lesser extent the optical gap $E_{\mathrm{g}}$, showed significant variations from one sample to another. Figure 7 shows the complex optical dispersion curves of the different $\mathrm{TiO}_{2}$ layers as computed from the best fits and compared with the reference dispersions of crystalline anatase $^{30,31}$ and rutile. ${ }^{32}$ Apart from the 18-nm thick nanolayer, all dispersions are typical of amorphous $\mathrm{TiO}_{2}$. Interestingly enough, the experimental data are well reproduced in the transparency region, especially in the infrared range, although the Tauc-Lorentz model imposes an imaginary part of the optical index to be zero below the gap energy. Such good agreement could not be reached if any defect in the deposited layer would have induced effective absorption or light diffusion and is, therefore, a sign of the excellent and industrially relevant optical quality of the layers obtained by the IAD process.
The main feature is the relatively important dependence of the optical index of the deposited layers on their actual thickness. Figure 8 shows this thickness dependence of the optical index (extracted at the 633-nm wavelength) and of the optical bandgap.

Clearly, two regimes are evidenced: first for layers thinner than $100 \mathrm{~nm}$, the thickness dependence of the optical index is very high and is associated to a rather high value of the bandgap compared with other ellipsometric studies, ${ }^{27,28}$ but comparable to other values obtained by the Urbach tail method. ${ }^{33}$ Second, above this threshold value, the index becomes thickness independent while the bandgap energy decreases. Such decrease of the bandgap has been associated by some authors to an improvement of the crystallinity of the films. ${ }^{10,16,26}$ In our case, the films have not been annealed and remain amorphous. However, a local and partial reordering of the $\mathrm{TiO}_{2}$ film cannot be excluded for the largest thicknesses.

\section{Application to the Design and Realization of a Complex Filter}

The ellipsommetric study discussed in the preceding section shows that the optical index of the $\mathrm{TiO}_{2}$ thin films deposited by the IAD process is in a first approximation constant if the

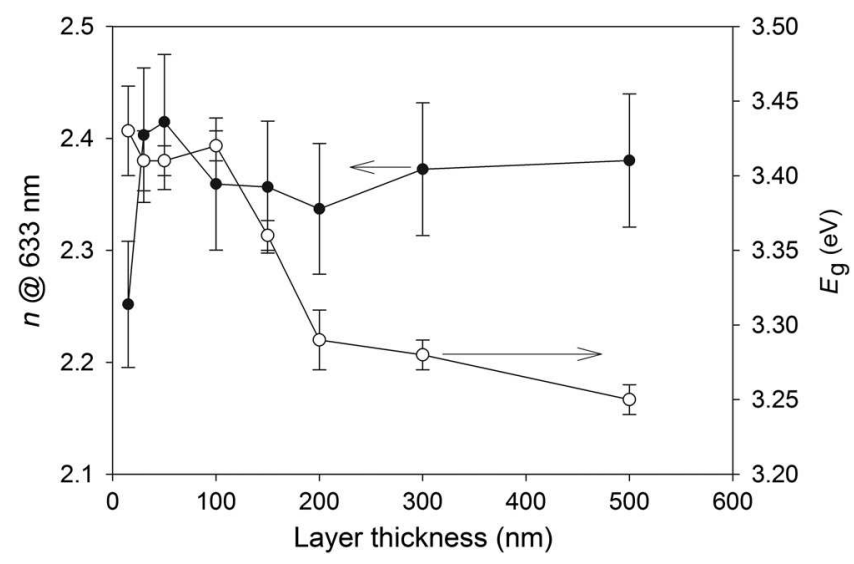

Fig. 8 Thickness dependence of the optical index (extracted at the $633-\mathrm{nm}$ wavelength) and of the optical band gap. 


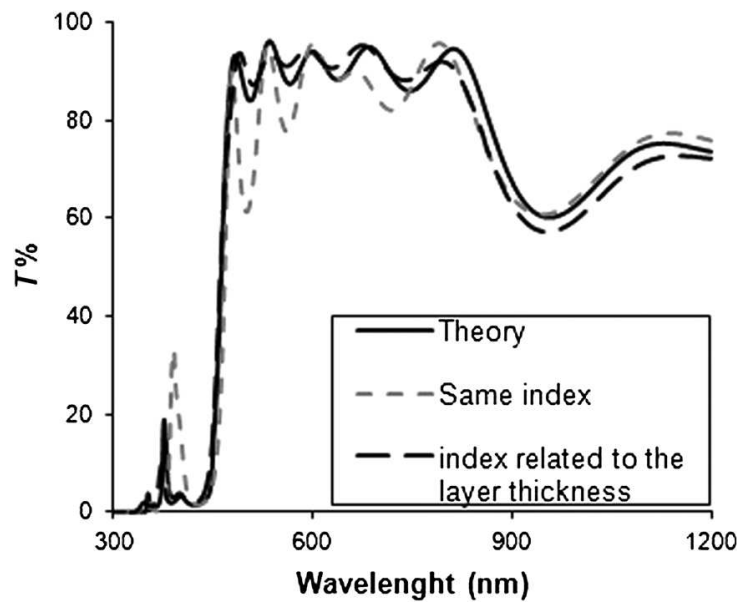

Fig. 9 Illustration of the incidence of the refractive index related to the thickness on an optical filter of 16 layers.

layer thickness falls in the range 100 to $500 \mathrm{~nm}$. This is a clear advantage for complex optical filters design, as the index of the layer is a crucial parameter for the optimization of the multilayer structure: if the optical index of a material is stable, then the realization will be robust to small fluctuations in the sample environment and deposition conditions.

This favorable situation does not hold any more for low thickness layers (between 15 and $100 \mathrm{~nm}$ ), for which the optical index varies of c.a. $7 \%$. This relative variation is large enough to strongly alter the final optical transmission of a complex multilayer filter that must contain nanolayers.

In order to illustrate this point, an optical filter has been designed using the standard iterative methods, ${ }^{34,35}$ as implemented in the commercial software Essentials Macleod (Thin film center Inc., Tucson, Arizona). This filter was optimized for a first stage of a sharp edge device transparent in the visible and NIR spectral ranges. It includes 16 dielectric layers alternating $\mathrm{SiO}_{2} / \mathrm{TiO}_{2}$ materials. Two alternative designs have been produced: one assuming an index of $\mathrm{TiO}_{2}$ independent of the thickness, and a second taking into account the variation of the optical index with the layer thickness according to the data of Fig. 7. Both filters have then been realized by IAD.

Figure 9 shows the comparison of the experimental filters transmission curves with the targeted theoretical one. Obviously, the transmission curve of the filter realized by taking into account the variation of the optical index with the layers' thicknesses (short dashed line) is much closer to the theoretical transmission (solid line). We interpret this very high sensitivity of the final transmission to the variation of the index by the determinant contribution of the nanosized layers to the adsorption edge of the filter. These low thickness films are the ones that show the largest variation of the optical index, resulting in a strong influence near the cut-off wavelength.

\section{Conclusions}

In this study, we found that the microstructure and the refractive index of a $\mathrm{TiO}_{2}$ monolayer depend strongly on the thickness below $100 \mathrm{~nm}$ and are really determined by the result of optical filters. Under these evaporation conditions, the AFM characterizations show that the layers are not rough. The
SEM and VASE show that the layers are dense and compact. This behavior is similar to another chamber with different conditions like the evaporation rate. The physical model used clearly expressed a nonlinear variation of the refractive index with the thickness of $\mathrm{TiO}_{2}$ and a high impact on the optical properties. The achievement of high quality optical devices requires consideration of this result.

\section{Acknowledgments}

SEM observations were performed at CMEBA at University of Rennes 1. Thanks to Professor J.C. Sangleboeuf, Director of LARMAUR Laboratory, for giving access to AFM equipment. Ellipsometry's measurements were carried out at IPR at University of Rennes 1 . Monolayers and filter were realized in vacuum chamber at Kerdry.

\section{References}

1. O. S. Heavens, "Thin-film optical filters," Opt. Acta Int. J. Opt. 33, 1336-1336 (2010).

2. M. Yamada and Y. Yamane, "A design method for dielectric multilayer optical filters," Opt. Rev. 3, A512-A517 (1996).

3. H. A. Macleod, Thin-Film Optical Filters, 4th ed., p. 800, CRC Press, Cleveland, Ohio (2010).

4. N. Démarest, "Développement d'empilements métalo-diélectriques pour des applications optiques: conception, réalisation et caractérisation," $\mathrm{PhD}$ thesis, University of Rennes 2013, http://hal.archives-ouvertes .fr/tel-00992394/.

5. L. I. Epstein, "The design of optical filters," J. Opt. Soc. Am. 42, 806 (1952).

6. C. Bundesmann et al., "Stress relaxation and optical characterization of $\mathrm{TiO}_{2}$ and $\mathrm{SiO}_{2}$ films grown by dual ion beam deposition," Thin Solid Films 516, 8604-8608 (2008).

7. M. R. Mohammadi and D. J. Fray, "Semiconductor $\mathrm{TiO}_{2}-\mathrm{Ga}_{2} \mathrm{O}_{3}$ thin film gas sensors derived from particulate sol-gel route," Acta Mater. 55, 4455-4466 (2007).

8. C. Lu and Z. Chen, "High-temperature resistive hydrogen sensor based on thin nanoporous rutile $\mathrm{TiO}_{2}$ film on anodic aluminum oxide," Sens. Actuators, B. 140, 109-115 (2009).

9. S.-H. Jeong et al., "Characterization of $\mathrm{SiO}_{2}$ and $\mathrm{TiO}_{2}$ films prepared using RF magnetron sputtering and their application to anti-reflection coating," Vacuum 76, 507-515 (2004).

10. P. Eiamchai et al., "A spectroscopic ellipsometry study of $\mathrm{TiO}_{2}$ thin films prepared by ion-assisted electron-beam evaporation," Curr. Appl. Phys. 9, 707-712 (2009)

11. C. H. Heo, S. B. Lee, and J.-H. Boo, "Deposition of $\mathrm{TiO}_{2}$ thin films using RF magnetron sputtering method and study of their surface characteristics," Thin Solid Films 475, 183-188 (2005).

12. M. Vergöhl et al., "Ex situ and in situ spectroscopic ellipsometry of MF and DC-sputtered $\mathrm{TiO}_{2}$ and $\mathrm{SiO}_{2}$ films for process control," Thin Solid Films 351, 42-47 (1999).

13. S. Murugesan et al., "Pulsed laser deposition of anatase and rutile $\mathrm{TiO}_{2}$ thin films," Surf. Coat. Technol. 201, 7713-7719 (2007).

14. P. Chrysicopoulou et al., "Optical properties of very thin $(<100 \mathrm{~nm})$ sol-gel TiO 2 films," Thin Solid Films 323, 188-193 (1998).

15. J. Aarik et al., "Influence of structure development on atomic layer deposition of $\mathrm{TiO}_{2}$ thin films," Appl. Surf. Sci. 181, 339-348 (2001).

16. M. Sreemany and S. Sen, "A simple spectrophotometric method for determination of the optical constants and band gap energy of multiple layer $\mathrm{TiO}_{2}$ thin films," Mater. Chem. Phys. 83, 169-177 (2004).

17. Y. Yamada et al., "Low temperature deposition of $\mathrm{TiO}_{2}$ films with high refractive indices by oxygen-radical beam-assisted evaporation combined with ion beam," Vacuum 66, 347-352 (2002).

18. C. Yang et al., "Effects of depositing temperatures on structure and optical properties of $\mathrm{TiO}_{2}$ film deposited by ion beam assisted electron beam evaporation," Appl. Surf. Sci. 254, 2685-2689 (2008).

19. D. Bhattacharyya et al., "Spectroscopic ellipsometry of $\mathrm{TiO}_{2}$ layers prepared by ion-assisted electron-beam evaporation," Thin Solid Films 360, 96-102 (2000).

20. T. Harada et al., "Influence of substrate surface roughness on light scattering of $\mathrm{TiO}_{2}$ optical thin films," Chin. Opt. Lett. 11, 10303 (2013).

21. Z.-J. Xu et al., "Thickness dependent optical properties of titanium oxide thin films," Appl. Phys. A 113, 557-562 (2013).

22. M. Sreemany and S. Sen, "Influence of calcination ambient and film thickness on the optical and structural properties of sol-gel $\mathrm{TiO}_{2}$ thin films," Mater. Res. Bull. 42, 177-189 (2007).

23. J. Rodríguez et al., "Thickness dependence of the optical properties of sputter deposited Ti oxide films," Thin Solid Films 365, 119-125 (2000). 
24. P. H. Tuan, Physics and Engineering of New Materials, Vol. 127, pp. 220-224, Springer, Berlin, Heidelberg (2009).

25. P. H. Smith, "A theoretical and experimental analysis of the ellipsometer," Surf. Sci. 16, 34-66 (1969).

26. X. Cheng et al., "Structure and properties of $\mathrm{TiO}_{2}$ films prepared by ion beam assisted deposition," Surf. Coat. Technol. 201, 5552-5555 (2007).

27. $\mathrm{D}$. $\mathrm{Li}$ et al., "Spectroscopic ellipsometry analysis of $\mathrm{TiO}_{2}$ films deposited by plasma enhanced chemical vapor deposition in oxygen/ titanium tetraisopropoxide plasma," Thin Solid Films 522, 366-371 (2012).

28. D. $\mathrm{Li}$ et al., "In situ spectroscopic ellipsometry study of $\mathrm{TiO}_{2}$ films deposited by plasma enhanced chemical vapour deposition," Appl. Surf. Sci. 283, 234-239 (2013).

29. G. E. Jellison and F. A. Modine, "Parameterization of the optical functions of amorphous materials in the interband region," Appl. Phys. Lett. 69, 371 (1996).
30. J. P. Borgogno, B. Lazarides, and E. Pelletier, "Automatic determination of the optical constants of inhomogeneous thin films," Appl. Opt. 21, 4020-4029 (1982).

31. G. E. Jellison et al., "Spectroscopic ellipsometry of thin film and bulk anatase (TiO[sub 2])," J. Appl. Phys. 93, 9537 (2003).

32. J. R. Devore, "Refractive indices of rutile and sphalerite," J. Opt. Soc. Am. 41, 416 (1951).

33. M. El-Raheemand and A. Al-Baradi, "Optical properties of as-deposited $\mathrm{TiO}_{2}$ thin films prepared by DC sputtering technique," Int. J. Phys. Sci. 8, 1570-1580 (2013).

34. A. Thelen, "Equivalent layers in multilayer filters," J. Opt. Soc. Am. 56, 1533 (1966).

35. C. Dufour and A. Herpin, "Application des Méthodes Matricielles au Calcul D'ensembles Complexes de Couches Minces Alternées," Opt. Acta Int. J. Opt. 1, 1-8 (1954).

Biographies of the authors are not available. 